\title{
Reproducing the Hip Anatomy: Intraoperative Planning and Assistive Devices (CAS, Robotics)
}

\author{
Marius Dettmer, Stefan W. Kreuzer, \\ and Stefany Malanka
}

\subsection{What Is the Rationale for Computer-Assisted Hip Arthroplasty?}

Total hip arthroplasty (THA) is a very successful procedure with $95 \%$ survivorship after 10 years and $80 \%$ survivorship at 25 years [1]. Despite this success, there are also reports about patient dissatisfaction, (early) revisions, and other issues.

Research has shown that appropriate femoral and acetabular component positioning and placement is crucial for prevention of hip dislocations, accelerated wear, leg length inequality, unfavorable biomechanics, and suboptimal function. One of the persisting challenges of appropriate acetabular component placement is its dependence on correct evaluation of the individual lumbopelvic kinematics and spine-hip relation.

Over the past decades, there have been multiple innovative approaches to improve positioning and placement of components to enhance alignment or to recreate native characteristics of the hip and femur, such as intraoperative fluoroscopy

M. Dettmer · S. Malanka

Memorial Bone \& Joint Research Foundation, Houston, TX, USA

e-mail: mdettmer@mbjc.net; SMalanka@mbjc.net

S. W. Kreuzer $(\bowtie)$

Memorial Bone \& Joint Research Foundation, Houston, TX, USA

Inov8 Orthopedics, Houston, TX, USA

e-mail: stefan@inov8hc.com, fcheney@inov8hc.com and mechanical navigation [2] technology. Potentially, the most influential innovation related to efforts in improving implant positioning was the introduction of computer-assisted surgery (CAS), built on the technological foundations and innovation and advances in major fields such as computing and optics.

When defining CAS, often computer technology for planning, navigating, and guiding surgery and the use of robotic assistance in surgery are used interchangeably to describe the term. While the fields of robotic assistance and computerassisted navigation are heavily intertwined and most robotics currently rely on computers and image-based preplanning for navigation (an exemption being the imageless NAVIO robotic system for knee arthroplasty by Smith \& Nephew, LPC), the underlying methods and techniques are distinct.

CAS systems comprise of a number of different technologies and methods to overcome challenges posed by arthroplasty. In total hip arthroplasty, CAS tracks the intraoperative position and alignment of the pelvis, femur, and surgical instruments. Orthopedic surgery may specifically benefit from this development, since bone matter is a great candidate for such measures due to its relative rigidity and its distinction from soft tissue in the body. For THA, CAS allows for accurate and appropriate placement of the acetabular component within the "safe zone" [3] and for recreation of the native femoral offset and leg 
length. Some devices further provide information about joint biomechanics, surgery progress, joint irregularities, and cutting accuracy [4].

\subsection{Imageless and Image-Based CAS}

A number of established navigation systems rely either on accelerometer-based tools or position/ motion capture technology that combines infrared cameras and reflective (passive) or light-emitting (active) markers or diodes attached to arrays/platforms, bony landmarks, and surgical tools. Software is then used to determine the position and orientation of the bone structures and instruments in $3 \mathrm{D}$ space for monitoring and provision of feedback. Imageless CAS includes an intraoperative bone registration process, where the identification and digitization of bony landmarks are crucial for developing a 3D model of the hip and to establish femoral position and orientation.

After registration and definition of planes, e.g., anterior pelvic plane, reaming depth/direction, and implant placement can be intraoperatively planned and modified. For image-based CAS, CTs or MRI is used for 3D modeling and subsequent preoperative planning, with intraoperative flexibility to modify the plan.

\subsection{Benefits, Complications, and Specific Risks of CAS}

Overall, both imageless and image-based computer navigation systems in THA are considered reliable and accurate. For imageless navigation, one study showed about $97 \%$ of acetabular components were placed within the safe zone for inclination and anteversion. A meta-analysis including 7 clinical trials and 485 patients compared THA with and without and imageless navigation, where desired position of anteversion deviated on average less in navigated cases and the authors found no differences in mean cup inclination and anteversion [5].

Leg length equality may be well restored with CAS with decreased outliers regarding leg length discrepancies, but there is no current scientific evidence that CAS may be superior regarding this aspect. There is also currently no evidence for significantly higher or specific risks related to CAS; results from a retrospective, small cohort study showed no differences after 5-7 years postop evaluation regarding clinical outcomes (HOOS, HHS, range of motion), bone mineral density, or polyethylene wear when comparing navigated and non-navigated THA [6]. Imagebased CAS has also been shown to be a valuable alternative to conventional THA, with highly precise and favorable measurements of cup alignment, less placement outside the safe zone, less dislocations, and similar survival rates [7].

A potential disadvantage of imageless navigation is the reliance on consistently accurate registration of bony landmarks for evaluation of the anterior pelvic plane (APP). The individual variability of soft tissue thickness overlaying landmarks such as the bilateral ASIS and the symphysis pubis may be challenging and lead to registration errors affecting cup positioning. It has been questioned whether the APP derived from the position of aforementioned landmarks as an anatomical reference plane for navigation may actually be inferior (especially in cases with difficulty to access the bony landmarks due to surrounding soft tissue) to the alternative supine coronal plane, which some systems allow to assess and use as a functional reference plane.

\subsection{Cost-Effectiveness of CAS}

Despite numerous positive reports regarding the safety, accuracy, and clinical outcomes, most surgeons have not yet adopted the technology citing high cost, the learning curve, and increased operative time. However, the complexity of current systems has significantly decreased throughout the past decade, and it allows for easier integration into the operating room (OR) workflow. Overall, there is a significant initial setup cost associated with the integration of computer navigation in the OR, so it has been postulated that lower priced systems may be crucial to justify setup and use of the technology for many facilities having to bal- 
ance cost and efficiency [8]. This initial setup cost may be well compensated by the longer term efficacy; overall, there may be a smaller number of required instrumentation trays [1], and implants, and a decrease of other indirect costs. Other major factors determining cost effectiveness may be the overall case volume (with decreased efficiency associated with lower number of surgeries), efforts to decrease revision rates in comparison to existing methods, and reducing costs of the technology itself, additional equipment, and disposables. The use of image-based navigation requires additional presurgery imaging, which further increases costs, and there has been some concern about infection due to pins placed percutaneously at the iliac crest, thus increasing risk for the patient. The latter has been identified and addressed by several navigation device manufacturers, as some devices either do not require the use of femoral trackers or use pinless technology where markers/trackers are attached to the limb surface without the need for incision or drilling. Alternatively, in certain systems, leg length may be acquired by positioning a probe on a distal femur landmark.

\subsection{Some Current Navigation Systems}

The imageless Intellijoint system (Intellijoint Surgical Inc., Waterloo, Canada) was developed to tackle current issues associated with conventional CAS, mainly the high per-patient costs, the increased surgical time, and the interruption of surgical workflow. This miniature tool acts as an intraoperative guidance tool that can provide information on cup position, leg length, offset, and hip center. The system is based on optical infrared technology described earlier and consists of a camera magnetically attached to a pelvic platform attached to the ipsilateral (contralateral in direct anterior approach arthroplasty) iliac crest with two screws, and a femoral disc tracking/registration point that is secured at the greater trochanter. The surgeon must also create an accurately reproducible tracking point at the distal femur, which can either be done via a small incision or some other surface marker that will not move.

This allows for a real-time evaluation of joint alignment and component positioning, and the magnetic array allows for easy adjustment of the camera-tracker setup, e.g., to attach the tracker to the bone impactor or a surgical probe. The native characteristics of the hip and femur are evaluated before dislocation; then during trial reduction, tracker measurements are conducted through the range of motion, and tracking then helps with selection of correct implant size and component placement (Fig. 10.1).

One major advantage of this system (apart from no requirements for additional imaging) is that the miniature-format system can be set up in the sterile field (with the camera sterilely draped and the monitor outside the sterile field) with no interruption of the surgical workflow. This also

Fig. 10.1 Intraoperative monitoring of leg length and offset using the Intellijoint miniature navigation system. The digitizing probe is used to trace the groove of the disc attached to the femur to establish femoral position and orientation changes

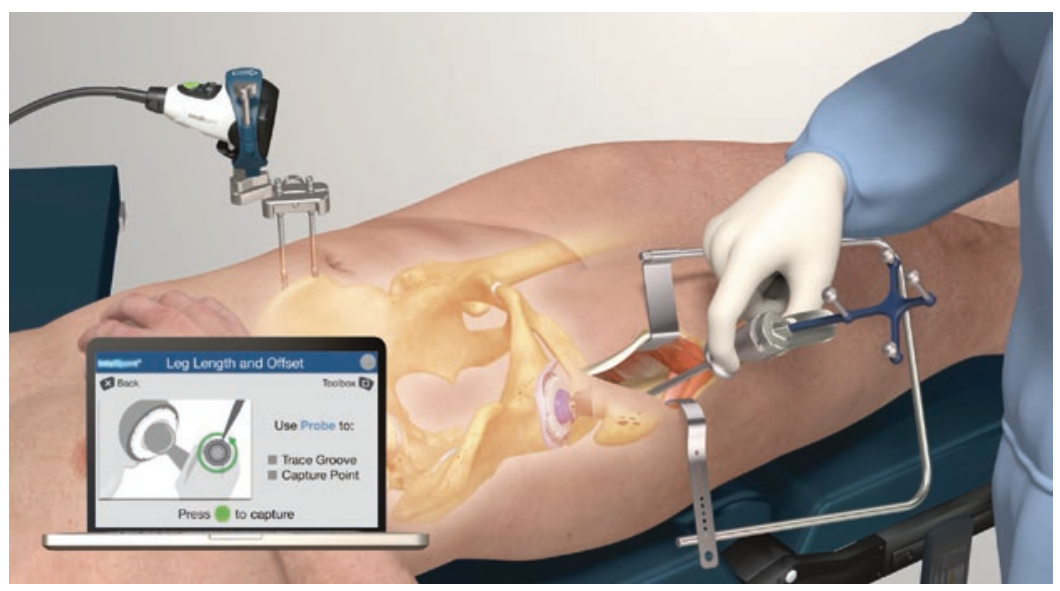


minimizes any issues regarding visibility of the markers for the camera.

The imageless Brainlab Hip System (Brainlab, Munich, Germany) uses wireless technology and is a touchscreen-based planning and navigation module. A single camera unit (consisting of two cameras for $3 \mathrm{D}$ space) that is part of the navigation station outside the sterile field emits and detects infrared flashes. Just as with other similar systems, markers that are attached to reference arrays on patients' bony landmarks (and a pinless femur reference option), probes, and instrumentation reflect the infrared light back to the camera system where it is detected and processed by software to calculate the 3D positions of the different landmarks and instruments. Preoperatively, the operator measures ASIS distance and pelvic tilt. Then, after making the incision and preparing the bone, a landmark registering process follows, which provides the computer with reference landmarks in space relative to reference arrays and information about individual patient anatomy. Instrument adapters allow for the use of devices not provided by the manufacturer, but require additional calibration steps. Intraoperative planning can be conducted in regards to both cup and femoral component, and "leg situation analysis" allows for intraoperative evaluation of leg length and combined femoral and pelvic offset (Fig. 10.2). It also allows for intraoperative range of motion and, depending on approach, impingement analysis.

Stryker's OrthoMap imageless system (Stryker Corporation, Kalamazoo, MI, USA) allows for registration in both supine and lateral position for different approaches, intraoperative evaluation of leg offset, leg length, ROM, and joint stability, and enables the user to intraoperatively plan both the cup and stem. Users may utilize the Stryker instruments or compatible instruments from several other cup manufacturers. A single infrared light-emitting and detecting unit is used in combination with markers/tracker attached to the pelvis and the femur, whereas leg length assessments can also be done without the use of a femoral tracker by using skin marker digitization at the distal femur. Cup positioning is based on the anatomical definition of cup alignment and allows for the determination of inclination, anteversion, and hip center shift (translation of the cup relative to the hip center in 3D space). Stem alignment and position can be assessed (e.g., anteversion, leg length, and femoral offset).
Fig. 10.2 The Brainlab system for THA uses a proximal femoral screw/ tracker that can be reacquired throughout the procedure for assessment of the leg length and offset

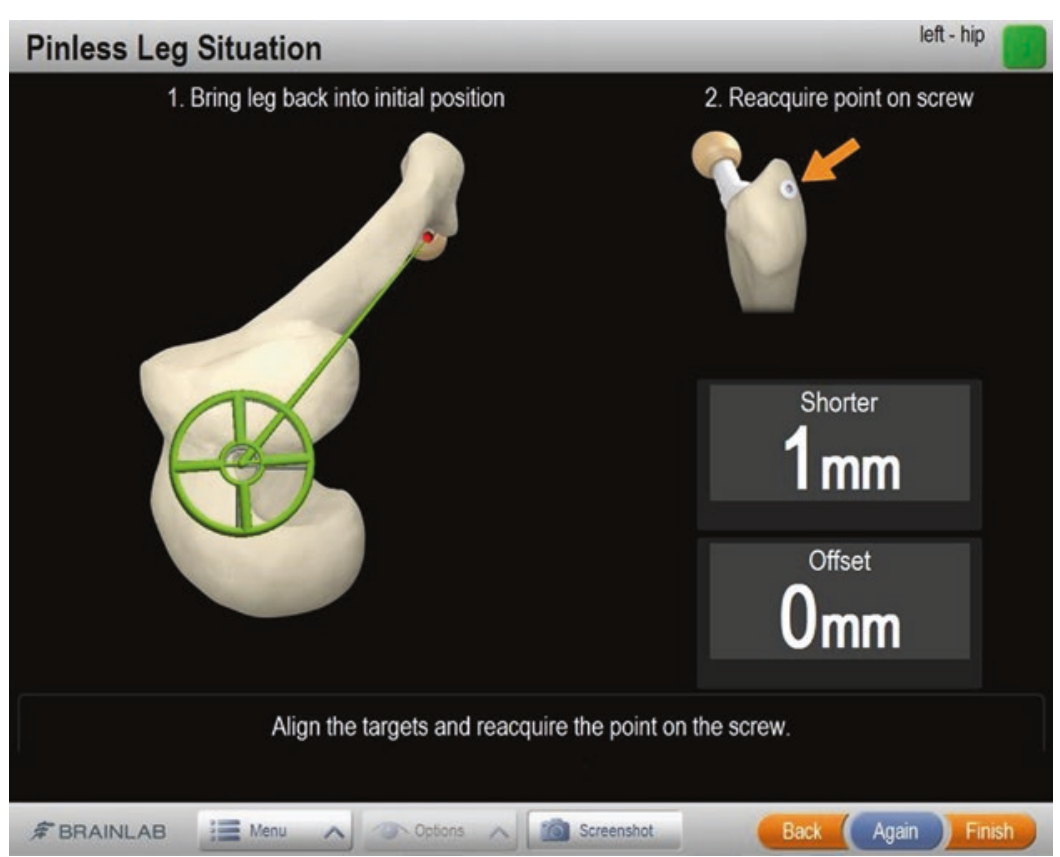




\subsection{Robotic Assistance}

It has been proposed that every industry, including modern medicine and ultimately total joint arthroplasty, follows a similar set of steps of maturation with the final phases being automation and computer integration [9]. Hence, the development of robotic assistance for orthopedic surgery is not surprising. Robotic assistance in joint surgery has been around since the 1990s, with William Bargar and Howard Paul developing the technique in 1992 for hip arthroplasty. Robotic devices such as the pioneering ROBODOC (Curexo Technology, Fremont, CA and Think Surgical Inc., Fremont, CA) have the robot navigate based on mechanical, computer-assisted navigation, and meticulous preplanning/templating before surgery.

Surgical robots can be classified as active, semi-active, and passive systems. Passive systems assist in parts of the surgery, under complete guidance from the surgeon, e.g., keeping a guide in position while the surgeon performs the bone preparation. Semi-active systems require intervention from the surgeon in form of manipulation of the cutting tool, while the system provides the operator with either haptic, visual, and/or auditory feedback regarding predetermined (preplanned) spatial constraints associated with the cutting process (i.e., "active constraint"). The Mako system (Stryker Corporation, Kalamazoo, MI, USA) is an approved and well-known semiactive system providing haptic guidance throughout the cutting procedure. Active robots execute tasks such as cutting without dependence on guidance from the surgeon, and once initiated, conduct the bone preparation autonomously.

The first robot of this kind was the aforementioned ROBODOC built on the platform of a traditional computer-assisted manufacturing system [10]. Another system is the TSolution One (Think Surgical, Inc., Fremont, CA), which is built on the foundation of ROBODOC technology. The overall strongest argument for the use of robotic assistance is the improved accuracy of bone milling in combination with 3D planning of the surgery for optimal bone preparation and implant placement.

\subsection{Benefits, Complications, and Specific Risks of Robotics}

Most of the existing studies comparing robotic surgery and traditional THA include only small cohorts, allowing only for preliminary conclusions about efficacy and safety. However, a recent meta-analysis of 178 articles (eight studies included in quantitative synthesis) and including only studies from 2005 to 2017 provided more insight regarding this topic [11]. Their analysis of intraoperative complications (femoral fractures/ cracks) and postoperative complications (infection, nerve palsy, deep vein thrombosis, and dislocation) showed a significantly higher intraoperative complication rate for manual THA and similar postoperative complication rates between manual and robotic-assisted THA. The total complication rates were significantly higher in manual THA, including in the three included randomized-controlled trials. There were no significant differences between the THA methods for several outcome measures (Japanese Orthopaedic Association Score, Harris Hip Score, Merle d'Aubigne Hip Score, Western Ontario and McMaster Universities Osteoarthritis Index). Radiographic data analyses showed no differences in leg length discrepancy, but a higher rate of optimal cup placement (within the safe zone) for robotic THA. This efficient and consistent placement within the margins of the safe zone is, in turn, associated with a decreased risk for dislocation, instability, and revisions. The authors found no significant differences regarding surgical time in the pooled analysis but an overall trend toward shorter surgery times for conventional THA. This factor may be related to each surgeon's individual experience and the learning curve, and more research is required to investigate how much surgeon experience can affect shortening of robotic surgery times. Blood loss was only evaluated in two studies, with one favoring robotic THA, and one finding no differences. Additionally, it has been suggested that there has been an increased number of litigations related to earlier robotic devices, potentially due to patients' perception that the use of robots indi- 
cates a lack of human oversight or decreased human control and involvement during the surgery [4].

Robots are designed to execute a plan, and a current obstacle or limitation is the inability of active robot systems to adjust this plan intraoperatively (intraoperative modifications are much less of an issue in semi-active robotic assistance THA), since there is a dynamic environment whose variables may change in an instant. For example, if there is an intraoperative finding that requires adjustments or if there is a fracture occurring, the surgeon has to stop the robot and manually finish bone cuts as there is no flexibility of the robotic technology at this present time in case of unforeseen events. This current problem of active robotics will most likely be solved in the future with progress in artificial intelligence and improved control software.

\subsection{Cost-Effectiveness}

A major issue regarding the current lack of cost efficiency is the high cost of purchasing the robotic system and its hardware and software components, and associated costs such as cost of disposables, training of staff, and maintaining the system, that is, (re-)calibrations, upgrades. Conventional THA has an advantage regarding intraoperative time as a major cost evaluation aspect, but with current developments in robotic technology, we can expect intraoperative time to further decrease, e.g., with development of simpler bone registration methods and improved OR workflow. Another very significant aspect are potential cost savings associated with robotic surgery related to the significant reduction of instruments needed and potential elimination of inventory in the field. To realize these cost savings, it will require a close collaboration between the individual stakeholders such as hospitals, OR facilities, device representatives, and physicians. Since there are still unanswered questions and a lack of long-term studies related to potential advantages/disadvantages of robotic THA, more research is needed to make valid statements about the overall cost-effectiveness. Ultimately, the market will decide whether the use of robotic technology is justified and the cost-effectiveness calculations will require constant updates and reevaluations, since the technology is still being further developed and refined.

\subsection{Personalizing Hip Replacement and the Role of CAS and Robotics}

The usefulness of Lewinnek's "safe zone" as a standard for cup orientation in THA has been questioned more and more in the past few years [12-14]. This is due to patients' individual muscular and bony anatomical differences that may affect lumbo-pelvic kinematics and spinehip relationship throughout the range of motion. These individual differences have been proposed to be crucial for finding accurate cup positioning to ensure optimal postoperative function and prevention of dislocation, instability, and premature failure. The general "safe zone" does not account for such individual differences, but there are efforts to take these aspects into consideration for THA. One promising approach is a preoperative evaluation of the patients' joint kinematics and lumbo-pelvic interactions, as provided by Corin's Optimized Positioning System (OPS, Corin, Cirencester, UK). The system uses preoperative imaging to investigate the individual dynamics of the lumbo-pelvic complex (Fig. 10.3). The kinematic information is then used to create an operative plan including suggestions for optimal cup inclination and anteversion (Fig. 10.4). The software also provides a preview of the planned osteotomy and the cup placement (Fig. 10.5). Such individualized systems for THA are likely to be useful for improving postoperative outcomes, but a solid created plan is only as good as its execution in the OR. For this specific system, Corin provides personalized jigs that are used to perform the suggested osteotomy/bone preparation on the acetabular and femoral side. 


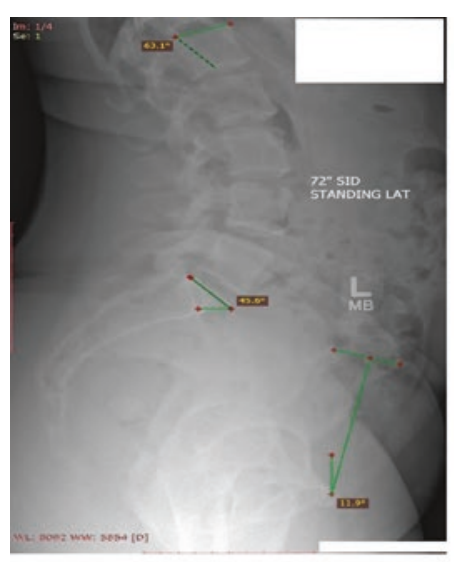

Standing parameters:

Pelvic tilt:

Sacral slope:

Lumbar lordotic angle:

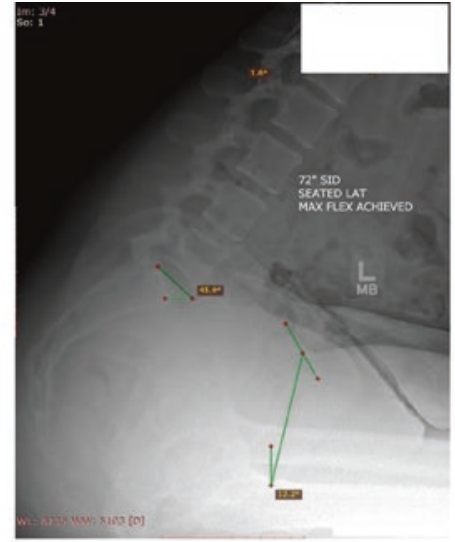

Flexed seated parameters:

Pelvic tilt:

Sacral slope:

Lumbar lordotic angle:

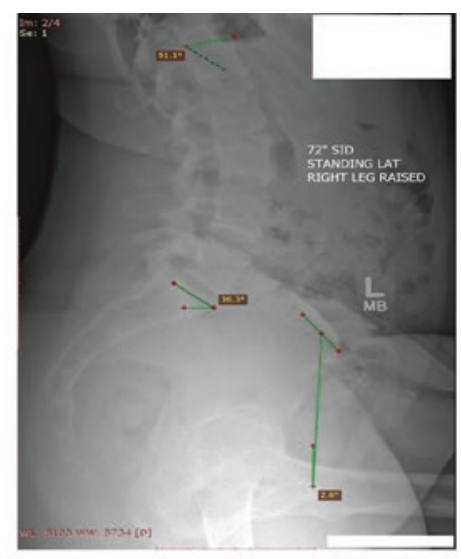

Step-up parameters:

Pelvic tilt:

Sacral slope:

Lumbar lordotic angle:

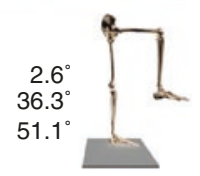

Fig. 10.3 Preoperative functional imaging for evaluation of individual kinematics and personalized cup orientation using the Optimized Positioning System (Corin OPS, Cirencester, UK)

Fig. 10.4 Operative plan for individualized cup orientation based on preoperative functional evaluation. (Corin OPS, Corin, Cirencester, UK)
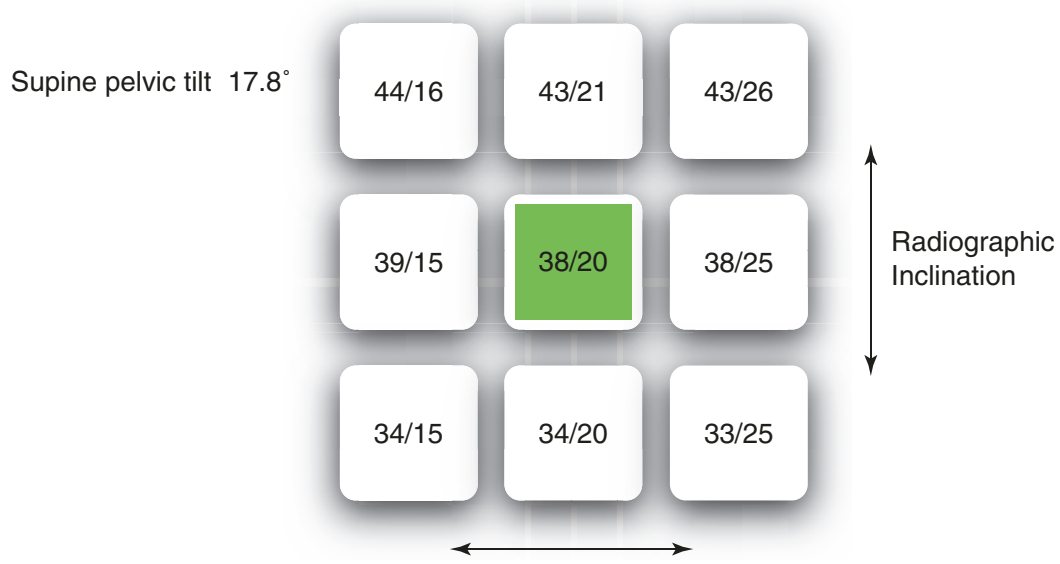

Radiographic Anteverion
However, for high-precision intraoperative navigation, the system could be combined and integrated with CAS, i.e., with the use of the Intellijoint system. This would allow the surgeon to use both a personalized approach through thorough preoperative planning in combination with high precision and accuracy operative execution with the help of CAS.

The value of this combination of preoperative planning and CAS may be further enhanced by concurrent developments in robotic assistance technologies. Hence, the functional assessment of native joint anatomy and kinematics in combination with CAS and the precision of robotic assisted bone preparation could lead to a paradigm shift in regards to THA standard of care; the technological progress and fine-tuned combination of the described tools could lead to significant improvements of orthopedic care. 


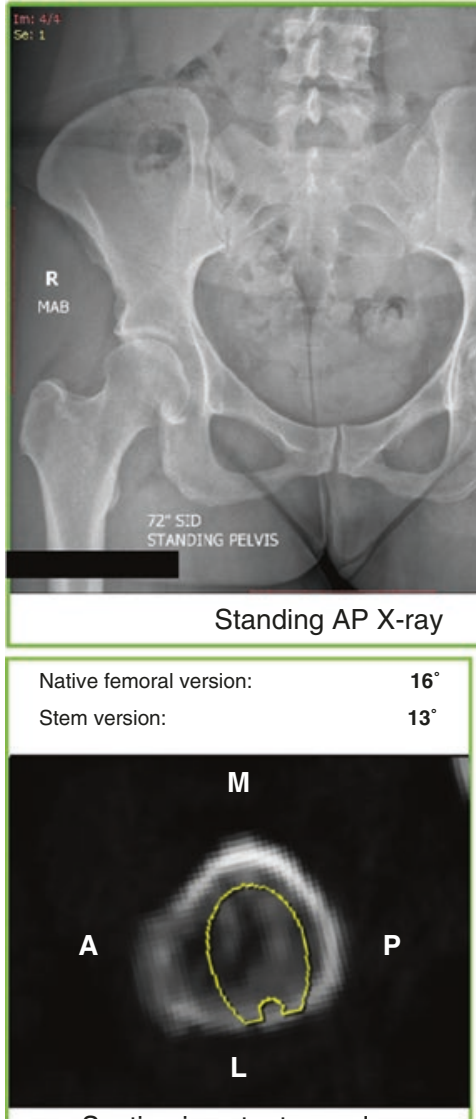

Section in osteotomy plane

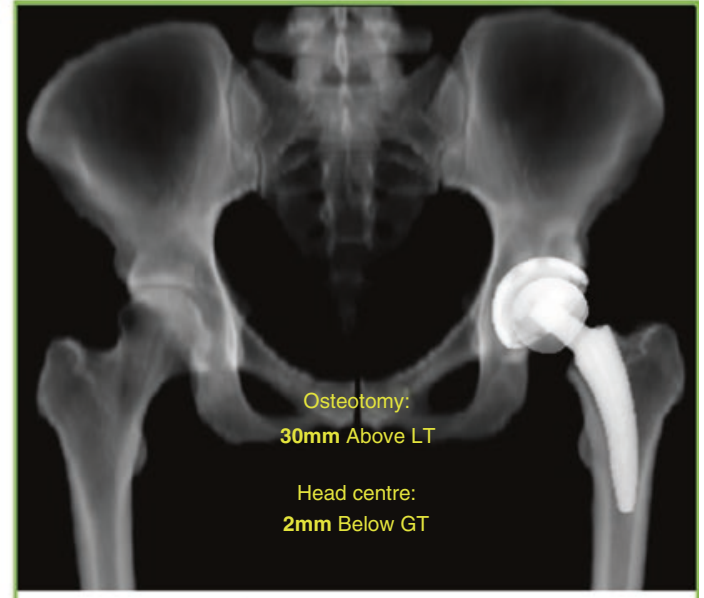

Supine post-operative plan
Cup orientation when supine: $\mathbf{3 8}^{\circ} / \mathbf{2 0}^{\circ}$

Cup: 50 Trinity

Sterm: \#3 MiniHip

Head: $36-4$

The default stem placement is planned to reproduce the native femoral head centre in the transverse plane, unless specified othenwise. See page 2 for details.

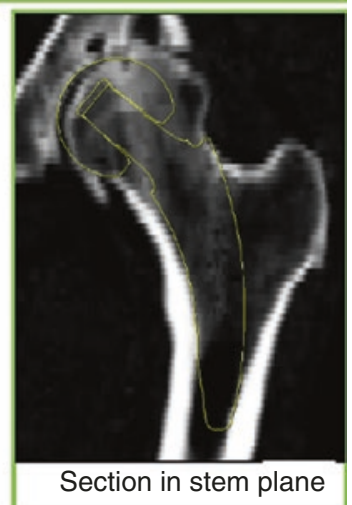

Fig. 10.5 Preview of planned osteotomy and cup placement with the Optimized Positioning System (OPS, Corin, Cirencester, UK)

\subsection{Future of CAS and Robotic Surgery}

"The only current limitation in the application and adoption of these technologies is the imagination and understanding of what can be accomplished in the future" [15]. Overall, the potential developments in CAS and robotic surgery could include improved preoperative and intraoperative planning and streamlined workflow, improved time efficiency, accuracy, and more flexibility. The aforementioned combination of preoperative functional hip analyses and operative plan execution may be beneficial to make THA more efficient and minimize the number of patients dissatisfied with their treatment. Navigated THA and robotic surgery have benefitted greatly from the technological advancements of the past two decades, specifically in the areas of computation, optical positioning/motion capturing, and industrial robotics. While it is difficult to predict the future, the past has indicated which path future innovations could take. On a sensor/imaging level, the use of ultrasound for defining a reference plane, or 3D laser scanning could become more important once the technologies are introduced in the workflow.

Whereas it is impressive how precisely robots perform their given tasks, future developments in artificial intelligence and sensor technology as 
part of the "Industry 4.0" phase may allow robots to be more efficient than they are currently; more advanced robots could be able to adjust to changing intraoperative variables and be able to perform modified bone cuts without requirements for intervention of the surgeon. Once robots will be able to distinguish between tissue types, they may assist both in soft tissue preparations and balancing and also be less likely to injure bonesurrounding ligaments, tendons, or blood vessels.

In their outlook regarding the future potential of robotic innovation, Jacofsky and Allen [9] suggest that a recreation of the former kinematics of the native joint with less emphasis on imaging will be a potential next step, and there are recently initiated efforts to develop highly sophisticated customized and personalized implants that potentially will not even be implantable without the use of robotic assistance.

Automation and robotics will not be excluded from future THA developments, and most likely play a larger role than today. "...one thing is clear: robotics appears to be here to stay" [9].

\section{Case Report}

This particular case was a 26-year-old Caucasian woman with a Crowe IV dysplastic hip and pseudo-acetabulum (Fig. 10.6), who reported

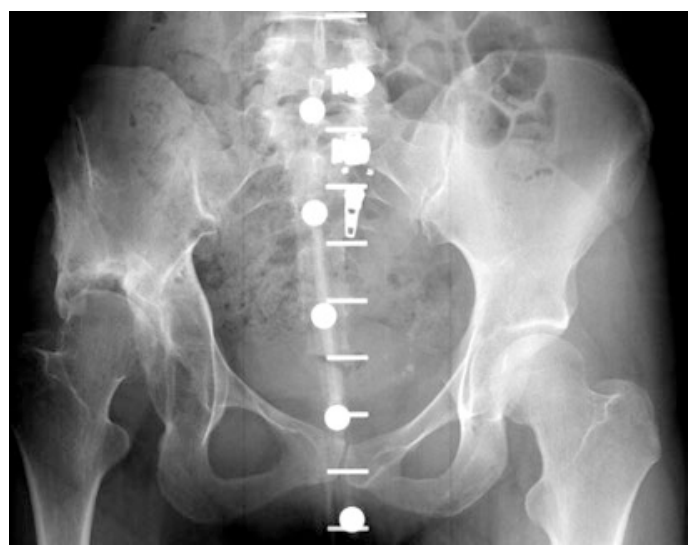

Fig. 10.6 Preoperative X-rays of 26-year-old patient with a Crowe IV dysplastic hip and pseudo-acetabulum having had symptoms since the age of 12 years. The patient had previously undergone Chiari pelvic osteotomy, femoral osteotomy, and femoral lengthening, which were unsuccessful in improving pain and function in the longer term.

Considering the history of the patient in terms of disease progression, pseudo-acetabulum, deformities, and earlier treatments, preoperative planning would have been extremely difficult without CAS and robotic assistance. The semiactive robotic assistance also allowed for intraoperative plan modifications regarding cup placement to recreate the true acetabulum and femoral preparation to reproduce the proximal femur anatomy. We used the MAKOplasty Robotic Arm Interactive Orthopedic (RIO) robotic arm (MAKO Surgical Corp., Ft. Lauderdale, FL, USA) and surgery-planning software, which allowed us to preoperatively plan the placement of the acetabular shell into the severely dysplastic true acetabulum based on patient-specific anatomical characteristics (based on three-dimensional [3D] reconstructions of patient computed tomography $[\mathrm{CT}]$ scans, Fig. 10.7). Also, a small femoral tracker was placed on the proximal femur and a small electrocardiogram (ECG) lead was attached to the kneecap, so the system's digitizer could be used to register those points for intraoperative evaluation of leg length and combined offset. This provided the opportunity to make intraoperative modifications when necessary. The use of the robotic arm helped to optimize accuracy and to execute the surgery plan with high levels of precision.

A Trinity shell (Corin, Cirencester, UK) was fixated using two screws and a neck-preserving MiniHip (Corin, Cirencester, UK) femoral stem was used. The surgery led to the reconstruction of the true acetabulum, which was associated with a decrease in leg length discrepancy (Fig. 10.8). The use of a neck-preserving short-stem implant in combination with use of the MAKO system helped to recreate the proximal femur anatomy. The patient followed up over 18 months and reported no issues related to her hip in the postoperative phase. 

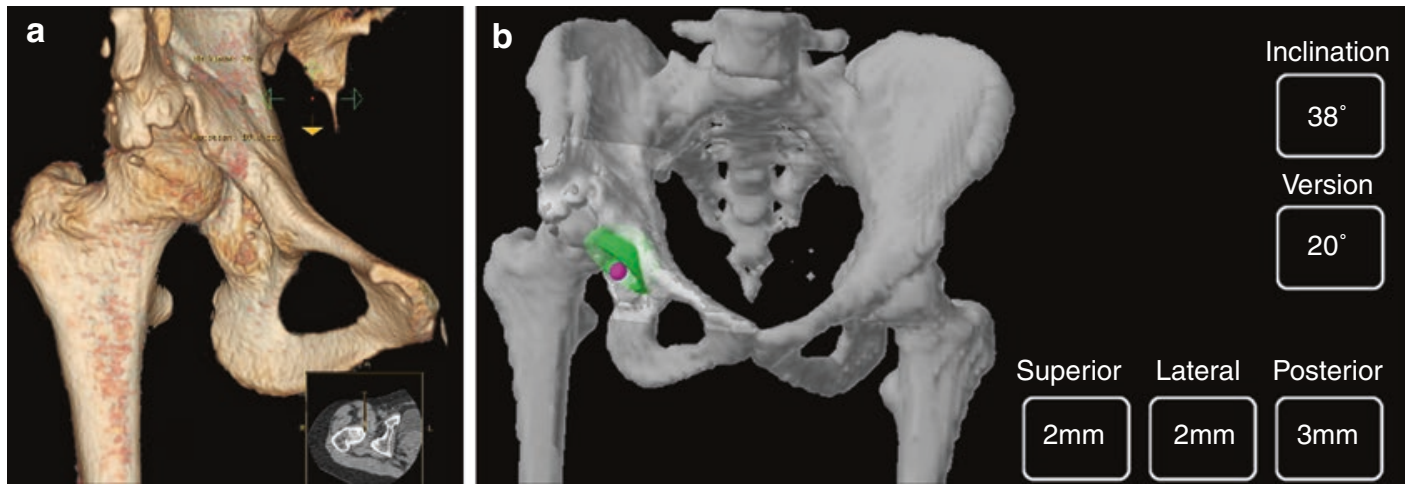

Fig. 10.7 (a) 3D model of the hip showing pseudo-acetabulum and (b) planned position of the shell in the dysplastic true acetabulum

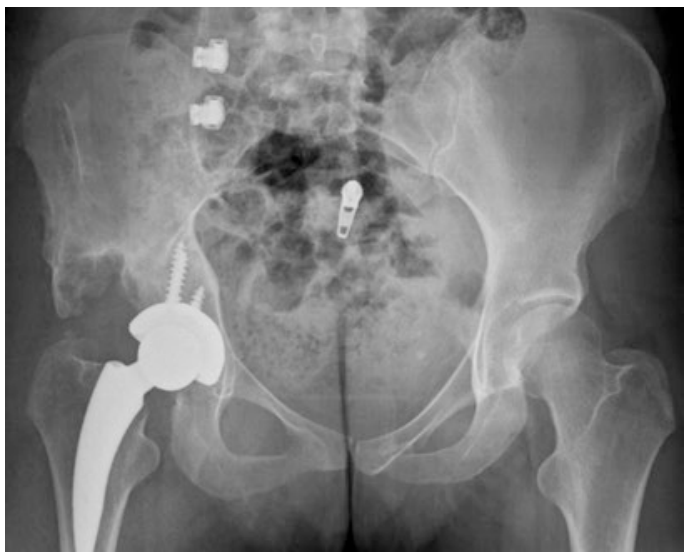

Fig. 10.8 Postoperative X-rays showing recreated true acetabulum and short-stem femoral implant

\section{References}

1. Deep K, Shankar S, Mahendra A. Computer assisted navigation in total knee and hip arthroplasty. Sicot J. 2017;3:50. http://www.sicot-j.org/10.1051/ sicotj/2017034.

2. Steppacher SD, Kowal JH, Murphy SB. Improving cup positioning using a mechanical navigation instrument. Clin Orthop Relat Res. 2011;469(2):423-8.

3. Lewinnek GE, Lewis JL, Tarr R, Compere CL, Zimmerman JR. Dislocations after total hip-replacement arthroplasties. J Bone Joint Surg Am. 1978;60(2):21720. http://www.ncbi.nlm.nih.gov/pubmed/641088.

4. Lang JE, Mannava S, Floyd AJ, Goddard MS, Smith BP, Mofidi A, et al. Robotic systems in orthopaedic surgery. J Bone Joint Surg Br. 2011;93(10):1296-9. http://www.ncbi.nlm.nih.gov/pubmed/21969424

5. Liu Z, Gao Y, Cai L. Imageless navigation versus traditional method in total hip arthroplasty: a metaanalysis. Int J Surg. 2015;21:122-7. https://doi. org/10.1016/j.ijsu.2015.07.707.
6. Keshmiri A, Schröter C, Weber M, Craiovan B, Grifka J, Renkawitz T. No difference in clinical outcome, bone density and polyethylene wear 5-7 years after standard navigated vs. conventional cementfree total hip arthroplasty. Arch Orthop Trauma Surg. 2015;135(5):723-30.

7. Waddell BS, Carroll K, Jerabek S. Technology in arthroplasty: are we improving value? Curr Rev Musculoskelet Med. 2017:378-87.

8. Inaba Y, Kobayashi N, Ike H, Kubota S, Saito T. The current status and future prospects of computerassisted hip surgery. J Orthop Sci. 2016;21(2):10715. https://doi.org/10.1016/j.jos.2015.10.023.

9. Jacofsky DJ, Allen M. Robotics in arthroplasty: a comprehensive review. J Arthroplasty. 2016;31: 2353-63.

10. Dungy DS, Netravali NA. Active robotics for Total hip arthroplasty. Am J Orthop. 2016;45(4):256-9.

11. Chen X, Xiong J, Wang P, Zhu S, Qi W, Peng H, et al. Robotic-assisted compared with conventional total hip arthroplasty: systematic review and metaanalysis. Postgrad Med J. 2018;2:335-41.

12. Abdel MP, von Roth P, Jennings MT, Hanssen AD, Pagnano MW. What safe zone? The vast majority of dislocated THAs are within the Lewinnek safe zone for acetabular component position. Clin Orthop Relat Res. 2016;474(2):386-91.

13. Tezuka T, Heckmann ND, Bodner RJ, Dorr LD. Functional safe zone is superior to the Lewinnek safe zone for total hip arthroplasty: why the Lewinnek safe zone is not always predictive of stability. J Arthroplast. 2018;34(1):3-8. https://doi. org/10.1016/j.arth.2018.10.034.

14. Reina N, Putman S, Desmarchelier R, Sari Ali E, Chiron P, Ollivier M, et al. Can a target zone safer than Lewinnek's safe zone be defined to prevent instability of total hip arthroplasties? Case-control study of 56 dislocated THA and 93 matched controls. Orthop Traumatol Surg Res. 2017;103(5):657-61.

15. DiGioia AM, Jaramaz B, Colgan BD. Computer assisted orthopaedic surgery. Image guided and robotic assistive technologies. Clin Orthop Relat Res. 1998;354:8-16. 
Open Access This chapter is licensed under the terms of the Creative Commons Attribution 4.0 International License (http://creativecommons.org/licenses/ by/4.0/), which permits use, sharing, adaptation, distribution and reproduction in any medium or format, as long as you give appropriate credit to the original author(s) and the source, provide a link to the Creative Commons license and indicate if changes were made.
The images or other third party material in this chapter are included in the chapter's Creative Commons license, unless indicated otherwise in a credit line to the material. If material is not included in the chapter's Creative Commons license and your intended use is not permitted by statutory regulation or exceeds the permitted use, you will need to obtain permission directly from the copyright holder. 